\title{
Juvenile Polyps: a large pediatric cohort
}

\author{
Victor L Fox*, Stephen Perros, Hongyu Jiang, Jeffrey Goldsmith \\ From 13th Annual Meeting of the Collaborative Group of the Americas on Inherited Colorectal Cancer \\ Honolulu, Hawaii, USA. 16-17 October 2009
}

\section{Background}

Juvenile polyps, classified as hamartomatous lesions with neoplastic potential, are the most common gastrointestinal polyp of childhood. Risk factors for neoplasia include germline DNA mutations, a family history of juvenile polyps, and multiple polyps ( $\geq 3$ or $\geq 5$ ). Only a few large pediatric series ( $>100$ patients) of patients with juvenile polyps have been reported, with limited data about repeat surveillance colonoscopy and the incidence of neoplasia. The primary aim of this study was to identify a large cohort of children with one or more juvenile polyps for descriptive analysis of patient demographics, polyp number, location, repeat colonoscopy, and neoplasia.

\section{Methods}

Juvenile polyp patients were identified by searching a single hospital pathology database from 1990-2009. Medical records were reviewed for each patient including demographics, family history, genetic testing, and colonoscopy and pathology reports noting polyp number, location, and histology. The right colon was defined as proximal to the splenic flexure.

\section{Results}

A total of 1,667 polyps were identified in 257 children, of which 158(61.5\%) were male. Median age at diagnosis was 5.6 yr (IQR: $3.7,8.8$ ). Germline DNA mutations were identified in 5 of $17(29.4 \%)$ patients tested including SMAD4 $(\mathrm{n}=2)$, BMPR1A $(\mathrm{n}=\mathrm{l})$, PTEN $(\mathrm{n}=2) .192$ patients underwent complete colonoscopy at initial diagnosis, revealing 1 polyp in 117(60.9\%)[Group A] and $>1$ polyp in $75(39.1 \%$ )[Group B]. 60(31.2\%) patients had $\geq 3$ polyps and $29(15.1 \%)$ patients had $\geq 5$ polyps. 128 (66.7\%) patients had polyps limited to the left colon and
$8(4.2 \%)$ patients had polyps limited to the right colon, 7 of which were single. Group B was more likely than Group A to have a family history of a 1st or 2nd degree relative with polyps (any type) or colon cancer $(\mathrm{p}=0.006)$ but no significant difference was found between groups for gender, age, or race. 62 of 192 (32.3\%) patients underwent repeat surveillance colonoscopy for polyp detection or removal. The number of times procedures were repeated were $2(\mathrm{n}=38), 3(\mathrm{n}=9), 4(\mathrm{n}=11)$, and 5,6 , $8,10(\mathrm{n}=\mathrm{l}$ for each). 44 of $75(58.7 \%)$ subjects with multiple polyps found at initial diagnosis underwent a second colonoscopy during the study period with a median time interval of 23.4 months (95\% CI: $14.2,41.5)$. Neoplasia was found in $4(1.6 \%)$ of 257 patients, three of which were male, including low grade dysplasia in 3 (ages 4.6, 6.9, and $14.6 \mathrm{yr}$ ), and adenocarcinoma with local metastases in one $11.8 \mathrm{yr}$ male without prior adenoma. Each of these patients had $\geq 5$ polyps detected during initial colonoscopy.

\section{Conclusions}

This largest series to date of children with juvenile polyps confirms previous observations regarding male predominance, early age of presentation, frequency of multiple polyps, and increased risk of neoplasia. The importance of complete colonoscopy is supported by finding isolated right colon polyps. Cancer may evolve in the absence of prior adenoma detection.

Published: 25 May 2010

doi:10.1186/1897-4287-8-S1-O2

Cite this article as: Fox et al:: Juvenile Polyps: a large pediatric cohort. Hereditary Cancer in Clinical Practice 2010 8(Suppl 1):O2.

\footnotetext{
*Correspondence: victor.fox@childrens.harvard.edu
Division of Gastroenterology and Nutrition, Department of Pathology, and
Clinical Research Program, Children's Hospital Boston, 300 Longwood Ave,

* Correspondence: victor.fox@childrens.harvard.edu
Division of Gastroenterology and Nutrition, Department of Pathology, and
Clinical Research Program, Children's Hospital Boston, 300 Longwood Ave,

* Correspondence: victor.fox@childrens.harvard.edu
Division of Gastroenterology and Nutrition, Department of Pathology, and
Clinical Research Program, Children's Hospital Boston, 300 Longwood Ave, Boston, Massachusetts 02115, USA
} 